\title{
Guidewire Compatibility
}

National Cancer Institute

\section{Source}

National Cancer Institute. Guidewire Compatibility. NCI Thesaurus. Code C150185.

The size of the guidewires that will fit through the inner lumen of the device. 